\title{
Peripheral vestibular disorders
}

\author{
Michael Strupp ${ }^{\mathrm{a}, \mathrm{b}}$ and Thomas Brandt ${ }^{\mathrm{b}, \mathrm{c}}$
}

\begin{abstract}
Purpose of review
First, to update the diagnosis, pathophysiology, and treatment of the most frequent peripheral vestibular disorders. Second, to identify those disorders for which the diagnostic criteria are still deficient and treatment trials are still lacking.

\section{Recent findings}

Bilateral vestibulopathy can be reliably diagnosed by the head-impulse test, caloric irrigation, and vestibular-evoked myogenic potentials. A new frequent subtype has been described: cerebellar ataxia, neuropathy, and vestibular areflexia syndrome. Benign paroxysmal positioning vertigo can be easily diagnosed and effectively treated. Vestibular neuritis is most likely caused by the reactivation of a herpes simplex type 1 infection; the inferior vestibular nerve subtype is now well established. More evidence is needed that the recovery can be improved by corticosteroids. Endolymphatic hydrops in Menière's disease can be depicted by high-resolution MRI after transtympanic gadolinium injection; a high-dosage and longterm prophylactic treatment with betahistine is evidently effective. Its mechanism of action is most likely an increase in the inner-ear blood flow. Vestibular paroxysmia is now a well established entity; carbamazepine is the treatment of first choice. Superior canal dehiscence syndrome can be reliably diagnosed; the best current treatment option is canal plugging.
\end{abstract}

\begin{abstract}
Summary
Although progress has been made in the diagnosis and treatment of most peripheral vestibular disorders, more state-of-the-art trials are needed on the treatment of bilateral vestibulopathy to prove the efficacy of balance training, of vestibular neuritis (in terms of recovery of peripheral vestibular function and central compensation), of vestibular paroxysmia to prove the effects of carbamazepine, and of Menière's disease to find the optimal dosage of betahistine.
\end{abstract}

\section{Keywords}

benign paroxysmal positioning vertigo, bilateral vestibulopathy, Menière's disease, vestibular neuritis, vestibular paroxysmia

\section{INTRODUCTION}

On the basis of several clinical studies, we are now able to correctly diagnose and treat the majority of peripheral vestibular disorders. Three typical forms of peripheral vestibular disorders can be differentiated by their characteristic signs and symptoms [1"]: chronic bilateral peripheral loss of vestibular function, characterized by oscillopsia during head movements and instability of gait and posture; acute/ subacute unilateral failure of vestibular function, characterized by a severe rotatory vertigo, oscillopsia, and imbalance; and paroxysmal, inadequate stimulation or inhibition of the peripheral vestibular system, characterized by attacks of vertigo and oscillopsia.

The six most frequent forms of peripheral vestibular disorders are - in the order of their frequency benign paroxysmal positioning vertigo (BPPV),
Menière's disease, vestibular neuritis, bilateral vestibulopathy, vestibular paroxysmia, and superior canal dehiscence syndrome (SCDS; Table 1) [1"].

Currently, the Classification Committee of the Bárány Society is defining and redefining the diagnostic criteria for these disorders [2]. This is important not only for daily clinical practice, but also for

${ }^{a}$ Department of Neurology, ${ }^{b}$ German Center for Vertigo and Balance Disorders and "Institute for Clinical Neuroscience, University Hospital Munich, Munich, Germany

Correspondence to Michael Strupp, MD, Professor of Neurology, Department of Neurology, German Center for Vertigo and Balance Disorders, University Hospital Munich, Campus Grosshadern, Marchioninistrasse 15, 81377 Munich, Germany. Tel: +49 897095 3678; fax: +49 897095 6673; e-mail: Michael.Strupp@med.uni-muenchen.de

Curr Opin Neurol 2013, 26:81-89

DOI:10.1097/WCO.0b013e32835c5fd4 


\section{KEY POINTS}

- On the basis of the anatomy, physiology, pathophysiology, and signs and symptoms, three types of peripheral vestibular disorders can be differentiated: chronic bilateral vestibular failure with the leading symptom of postural imbalance (bilateral vestibulopathy), acute unilateral vestibular failure with the leading symptom of acute onset rotatory vertigo (e.g., vestibular neuritis), and recurrent pathological excitation or inhibition of the peripheral vestibular system with the leading symptom of recurrent attacks of vertigo [e.g., BPPV, Menière's disease, vestibular paroxysmia, or superior canal dehiscence syndrome (SCDS)].

- The diagnosis of vestibular disorders is still based on the patient history and clinical examination, in particular the head-impulse test, positioning maneuvers, examination of eye movements, hearing tests, and Valsalva maneuver, as well as posture and gait, all of which often easily allow a precise diagnosis even without laboratory examinations.

- A new clinically relevant and frequent subtype of bilateral vestibulopathy was described which leads to a significant impairment of patients because of the combined deficits: cerebellar ataxia, neuropathy, and vestibular areflexia syndrome.

- In terms of the treatment, vestibular exercises are efficient in patients with vestibular deficits, liberatory maneuvers in BPPV (maneuvers depend on the affected posterior, horizontal, or anterior canal), corticosteroids probably in acute vestibular neuritis, betahistine and gentamicin in Menière's disease, carbamazepine probably in vestibular paroxysmia, and surgery in SCDS.

- There are still deficits or limited evidence in terms of the treatment, so we need further well designed placebocontrolled trials for acute vestibular neuritis, Menière's disease, and vestibular paroxysmia.

treatment trials. The various forms of vertigo and dizziness disorders can be treated with medical, physical therapy, surgery, and psychotherapeutic measures. The treatment of various peripheral vestibular disorders has been improved as a result of the findings of several controlled trials. There are still deficiencies, however, in the treatment of some these disorders, in particular bilateral vestibulopathy, Menière's disease, acute vestibular neuritis, vestibular paroxysmia, and superior canal dehiscence. In this overview, the major aspects of the cause, pathophysiology, clinical findings, diagnostic criteria, and the therapy of the above-mentioned six most frequent types of peripheral vestibular disorders are summarized.

\begin{tabular}{|c|c|c|}
\hline \multirow[b]{2}{*}{ Vestibular syndrome } & \multicolumn{2}{|c|}{ Frequency } \\
\hline & $n$ & $\%$ \\
\hline Benign paroxysmal positioning vertigo & 2618 & 17.7 \\
\hline Somatoform phobic postural vertigo & 2157 & 14.6 \\
\hline Central-vestibular vertigo & 1798 & 12.2 \\
\hline Vestibular migraine & 1662 & 11.2 \\
\hline Menière's disease & 1490 & 10.1 \\
\hline Vestibular neuritis & 1198 & 8.1 \\
\hline Bilateral vestibulopathy & 1067 & 7.2 \\
\hline Vestibular paroxysmia & 569 & 3.9 \\
\hline Other psychogenic vertigo & 453 & 3.1 \\
\hline Perilymph fistula & 83 & 0.6 \\
\hline Unclear vertigo syndromes & 408 & 2.8 \\
\hline Other disorders ${ }^{a}$ & 1287 & 8.8 \\
\hline Total number of patients & 14790 & \\
\hline
\end{tabular}

Data from Brandt et al. [1"].

${ }^{a}$ Other vertigo syndromes are, for example, nonvestibular vertigo in neurodegenerative diseases or nonvestibular ocular motor disorders in myasthenia gravis or peripheral ocular motor palsies.

\section{BILATERAL VESTIBULOPATHY}

Key symptoms are movement-dependent postural vertigo and unsteadiness of gait and (in $40 \%$ of patients) of the patients blurred vision when walking, as well as impaired spatial memory and navigation $[3,4]$. Bilateral vestibular failure is the most frequent cause of movement-dependent postural vertigo in elderly patients. The disease has a pronounced negative impact on physical and social functioning, leading to the deterioration of quality of life [5].

The diagnosis is confirmed by testing the vestibulo-ocular reflex (VOR) by a combination of a bilaterally pathological head-impulse test and the caloric test. These tests reveal three groups of patients in terms of canal function: those with a combined high- and low-frequency deficit (the majority), those with only a high-frequency deficit, or those with only a low-frequency deficit [6]. In addition, measurements of the dynamic visual acuity by either the VOR reading test to determine visual acuity during head turns [7] or during walking [8"] are also helpful to support the diagnosis. All these laboratory examinations test canal function [for tests on otolith function see the chapter on vestibular-evoked myogenic potentials (VEMPs) by 
Sally Rosengren]. There is also a rare subcategory of 'bilateral sacculopathy', in which the canal function is normal [9].

Up to $20 \%$ of patients with bilateral vestibulopathy frequently also have a cerebellar syndrome, in particular cerebellar ocular motor dysfunction and downbeat nystagmus $\left[10^{-"}, 11\right]$. This new syndrome is called cerebellar ataxia, neuropathy, and vestibular areflexia syndrome (CANVAS) [10"']. It is probably caused by neurodegeneration that affects the cerebellar, ocular motor, and vestibular systems with an additional neuropathy.

Treatment of the various forms of bilateral vestibulopathy follows three lines of action: prophylaxis of progressive vestibular loss, recovery of vestibular function, and promotion of central compensation or substitution [12] for missing vestibular function with physical therapy. The efficacy of balance training was confirmed at least for patients with unilateral peripheral vestibular function disorders [13] (see the chapter by Susan Herdman on vestibular rehabilitation). In the long term, vestibular implants will be a therapeutic option. They have had very promising results in animal studies [14] and also in a pilot trial in humans [15"'].

\section{VESTIBULAR NEURITIS}

The key signs and symptoms of vestibular neuritis are acute onset of sustained rotatory vertigo; horizontal rotatory peripheral vestibular spontaneous nystagmus toward the unaffected ear, which typically can be suppressed by visual fixation; a pathological head-impulse test; and postural imbalance.

The following aspects of vestibular neuritis will be discussed in the light of the latest literature: the differential diagnosis of vestibular neuritis and central vestibular pseudoneuritis, cause of the disease, subtypes of vestibular neuritis which affect the superior vestibular or the inferior vestibular nerve, long-term course, recurrence rate, complications, and causative treatment.

\section{Vestibular neuritis or vestibular pseudoneuritis}

From a clinical point of view, the first question to be answered in patients with acute vertigo and nystagmus is whether the symptoms are caused by vestibular neuritis or central 'vestibular pseudoneuritis'. Several clinical studies have been published on this issue over the last 5 years [16-20]. The following five signs (the big five) of a central lesion were identified: 'skew deviation' (vertical divergence) as a component of the ocular tilt reaction, gaze-evoked nystagmus contralateral to the quick phase of the spontaneous nystagmus, saccadic smooth pursuit, a normal head-impulse test in a patient with acute nystagmus, and a central fixation nystagmus, which is typically not suppressed by visual fixation. The combination of these clinical signs allows us to discriminate between a central and a peripheral lesion with a sensitivity and specificity of more than $95 \%$.

\section{Cause}

There is increasing evidence that vestibular neuritis is caused by the reactivation of a latent herpes simplex virus type 1 (HSV-1) infection: HSV-I DNA has been detected on autopsy with the use of polymerase chain reaction in about two of three human vestibular ganglia as well as the expression of CD8-positive T-lymphocytes, cytokines, and chemokines. These findings indicate that the vestibular ganglia are latently infected with HSV-1 [21]. Recent results of animal studies support this view: latently and lytically HSV-1 infection and reactivation were demonstrated in cultured rat vestibular ganglion cells [22]. A mouse model showed that the inoculation of HSV could induce vestibular dysfunction with infected vestibular ganglion cells [23].

\section{Superior and inferior vestibular nerve vestibular neuritis}

The superior vestibular nerve innervates the horizontal and anterior semicircular canals, the utricle, and the anterosuperior part of the sacculus. The inferior vestibular nerve innervates the posterior semicircular canal and the posteroinferior part of the sacculus. Three-dimensional recordings of spontaneous nystagmus and the dynamic VOR deficit showed that the function of the posterior canal is spared in most thus afflicted patients [24], that is, it most often affects the superior vestibular nerve. Measurements with ocular VEMP showed an impairment of the utricular function in most patients with acute vestibular neuritis [25], as well as of the saccular function by cervical VEMP $[26,27]$ (see the chapter by Sally Rosengren). This explains why patients with vestibular neuritis can suffer from posterior canal BPPV. Why is the superior vestibular nerve more often affected? It is longer and has more speculae [28]; further, the posterior semicircular canal is innervated by an additional anastomosis.

On the basis of the above-mentioned, threedimensional VOR measurements [24], the rarer subtype of inferior vestibular neuritis (accounting for about $10 \%$ of all patients with vestibular neuritis) has been examined in detail with three-dimensional vector analysis and in particular with cVEMPs and 
oVEMPs $[27,29,30]$. This is now the established cause of acute vertigo.

\section{Long-term course, recurrence rate, and complications}

In the course of the illness, the peripheral vestibular function does not spontaneously completely recover in most patients [31]. The recurrence rate is between 2 and $11 \%[32,33]$. In $10-15 \%$ of patients with vestibular neuritis, a typical BPPV develops in the affected ear within a few weeks [34]. It is likely that the otoconia loosen during the additional inflammation of the labyrinth, because HSV-1 DNA was also found in the labyrinth of humans and this eventually results in canalolithiasis. The second important complication is that vestibular neuritis can develop into phobic postural vertigo [35].

\section{Treatment}

The treatment of acute vestibular neuritis is based on three principles: first, symptomatic therapy for vertigo, nausea, and vomiting; second, causative treatment; and third, vestibular exercises to improve central compensation (for the latter, see the chapter by Susan Herdman).

Studies in the 1990s indicated that glucocorticoids can improve the course of 'acute vertigo'. A prospective, randomized, placebo-controlled study in 141 patients had shown that monotherapy with methylprednisolone significantly improved the peripheral vestibular function of patients [36]. Valacyclovir had no influence on the course of the disease, neither as monotherapy nor in combination with methylprednisolone. Although the benefit of steroids was confirmed by another study [37] as well as by a meta-analysis [38], a Cochrane analysis still makes no general treatment recommendation for corticosteroids. A trend was seen 1 month after the illness began [39]. More studies need to be performed, and the effects on quality of life have still not been investigated sufficiently [40]. This means that there is urgent need for at least two additional state-of-the-art clinical trials on the effects of steroids on the recovery of vestibular function and quality of life.

\section{BENIGN PAROXYSMAL POSITIONING NYSTAGMUS}

The definite diagnosis of benign paroxysmal positional vertigo (BPPV) requires positional maneuvers that result in a canal-specific positional nystagmus [41"]. The observance of this nystagmus allows one to differentiate between canalolithiasis of the posterior semicircular canal (pc-BPPV), the horizontal canal (hc-BPPV), and cupulolithiasis of the horizontal canal (hc-BPPV-cu). Further diagnostic classifications include canalolithiasis of the anterior canal (ac-BPPV), cupulolithiasis of the posterior canal (pc-BPPV-cu), lithiasis of multiple canals (mc-BPPV), as well as the distinction between 'probable' (spontaneously resolved) and 'possible' BPPV [41"]. Central cerebellar dysfunctions such as infarctions of the nodulus can mimic BPPV [42] or positional nystagmus; this is also found in vestibular migraine [43"].

Evidence-based reviews conclude that therapeutic positional maneuvers are most effective for the relief of typical BPPV, especially for canalolithiasis as compared to cupulolithiasis [44]. A new therapeutic maneuver has been described for ac-BPPV. It consists of sequential head positionings: beginning supine with head hanging $30^{\circ}$ downward with respect to the body, then supine with head inclined $30^{\circ}$ forward, and ending sitting with head inclined $30^{\circ}$ forward [45].

Patients with BPPV of the lateral semicircular canal can present with the geotropic form, in which the debris floats in the posterior arm of the canal (canalolithiasis), or with the apogeotropic form, in which the debris can either float in the anterior arm of the canal or be attached to the cupula (cupulolithiasis) [46]. Two different therapeutic positional maneuvers were tested for apogeotropic hc-BPPV in a randomized clinical trial: head-shaking with $3 \mathrm{~Hz}$ in the sitting patient while the head was held downward at an angle of $30^{\circ}$ and the so-called Gufoni maneuver, in which the patient lay on the ipsilesional side and turned his or her head upward to promote migration of the debris toward the vestibule [47"].

\section{MENIËRE'S DISEASE}

Menière's disease is clinically characterized by recurrent spontaneous attacks of vertigo, fluctuating hearing loss, tinnitus, and aural fullness. More than 6900 papers have been published on Menière's disease according to a PubMed search, and each year more than 100 new papers are published. This high number shows that there are still many areas of uncertainty about this disorder, although it was first described in 1861. Currently, two topics are being addressed in clinical research on Menière's disease: first, the imaging of the inner ear with high-resolution MRI after transtympanic gadolinium injection and second, the new treatment options (more than 3500 publications). 


\section{Visualization of endolymphatic hydrops}

Endolymphatic hydrops is the pathological hallmark of Menière's disease. In 2007, Nakashima et al. [48] were able to demonstrate endolymphatic hydrops in patients with Menière's disease in vivo by high-resolution MRI after transtympanic gadolinium injection, because gadolinium primarily diffuses into the perilymphatic space. Since then, this technique has been considerably improved, shown to be reliable [49], and has even been used for treatment trials to evaluate the effects of different agents on endolymphatic hydrops. The intravenous administration of gadolinium also causes an enhancement of the perilymphatic space but considerably less so than when given transtympanically $[50,51]$. Thus, the transtympanic route is currently favored. Vestibular and audiological deficits correlate with the extent of the endolymphatic hydrops [52-55]; moreover, a herniation of the membranous labyrinth into the horizontal semicircular canal could be correlated with impaired caloric response in Menière's disease patients. As regards the treatment and imaging, a case study on five patients reported that the outcome of a treatment with gentamicin could not be predicted by this imaging technique [56]. Another study with gentamicin in eight patients observed no change of endolymphatic hydrops in four and a worsening in three. Finally, a study on six patients treated with $48 \mathrm{mg}$ betahistine per day did not find a decrease of endolymphatic hydrops after a mean follow-up time of 11 months [57]. All in all, this is a very promising imaging technique for visualizing endolymphatic hydrops. It will considerably improve our knowledge of the pathophysiology and treatment of Menière's disease.

\section{Treatment}

There is a plethora of treatment strategies for Menière's disease: destructive procedures, effective but destructive; endolymphatic sac decompression, not effective [58]; restriction of salt and fluid intake and diuretics, also not effective [59]. Evidently, gentamicin, transtympanic glucocorticoids, and betahistine are effective [60].

\section{Gentamicin}

The effect of gentamicin is based on its direct damage to vestibular type 1 hair cells. Two prospective, double-blind, randomized controlled studies have shown efficacy for the symptoms of vertigo $[61,62]$. These results have been supported by a Cochrane analysis [63]. There are two fundamental problems with treatment with aminoglycosides. First, there is accompanying hearing damage, which affects at least $20 \%$ of the patients; for this reason, only patients with obvious preexisting hearing damage should actually receive this treatment. Second, it should be given only in one ear, although more than $40 \%$ of the patients develop a bilateral Menière's disease after a period of 20 years.

\section{Transtympanic administration of glucocorticoids}

A controlled, prospective, double-blind study showed that attacks of vertigo improved in $82 \%$ as opposed to $57 \%$ in the placebo group [64]. According to a Cochrane analysis [65], this study is so far the only one that has been performed with precise methods. Thus, there is only limited evidence that transtympanic administration of glucocorticoids is effective. In a prospective, controlled, randomized study, the effect of intratympanic gentamicin was compared with that of dexamethasone [66"]: gentamicin was clearly superior, reducing vertigo attacks by $93 \%$ compared with $61 \%$ with dexamethasone.

\section{Betahistine}

This agent is a weak histamine $\mathrm{H} 1$ agonist and a stronger H3 antagonist. Despite its widespread use, its pharmacological mode of action has not been entirely elucidated. Several animal studies have shown that betahistine increases cochlear blood flow in vivo; none of these studies, however, found a dose-response curve. The dosage of betahistine administered intravenously in these studies ranged from 0.1 to $10.0 \mathrm{mg} / \mathrm{kg}$ body weight. Corresponding doses for clinical use, however, range from about 0.05 to $0.6 \mathrm{mg} / \mathrm{kg}$ body weight orally $[67,68]$. Recently, the effects of betahistine on cochlear microcirculation at dosages corresponding to clinically used doses were reevaluated in an animal model [69"']. When betahistine was administered at a dose of $1.0 \mathrm{mg} / \mathrm{kg}$ body weight, cochlear blood flow was increased to a peak value of 1.34 arbitrary units compared to baseline with a sigmoid correlation between the increase in blood flow and the dosages (Fig. 1). The effects of the dosage range of betahistine on cochlear microcirculation corresponded well to the clinically used single dosages to treat Menière's disease. This suggests that the improved effects of higher doses of betahistine for the treatment of Menière's disease might be because of a corresponding increase of cochlear blood flow [69"'].

Meta-analyses show that betahistine evidently has a prophylactic effect on the attacks of Menière's disease [70], although no placebo-controlled, 


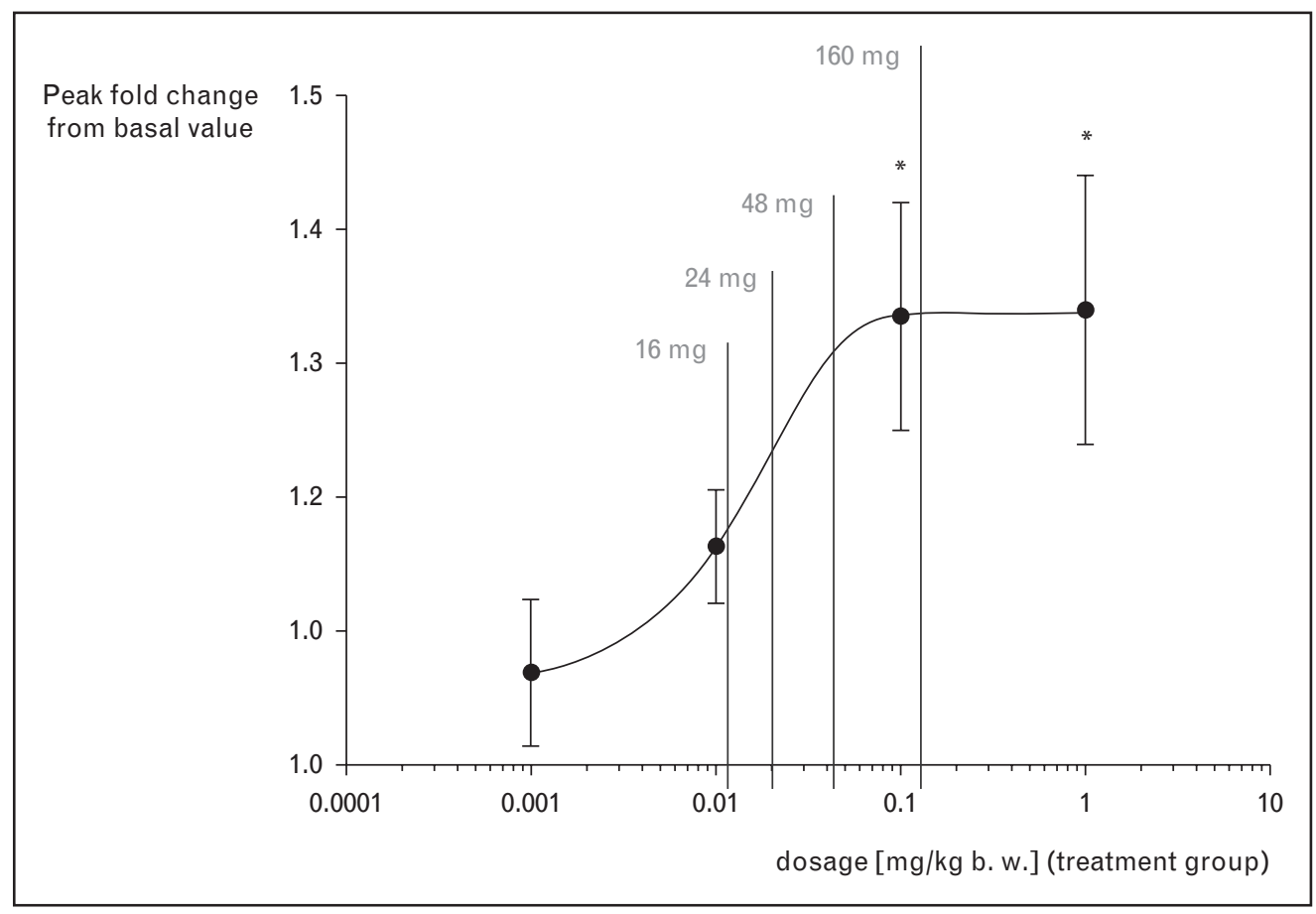

FIGURE 1. Peak values of cochlear blood flow with curve fitted by nonlinear regression and calculated corresponding oral dosage (peak mean $\pm S D ;{ }^{*} P<0.05$ ) (from [69-"]) showing a sigmoid increase of blood. This correlates with the increasing dosages currently used for the treatment of Menière's disease.

double-blind study has yet been published. On the basis of clinical experience with a dosing regimen of $3 \times 48 \mathrm{mg} /$ day betahistine dihydrochloride, an open pilot study was performed in 112 patients receiving either $3 \times 16$ or $3 \times 24 \mathrm{mg} /$ day or $3 \times 48 \mathrm{mg} /$ day. This study showed that the higher doses were significantly superior to the usual dosage of $3 \times 16-$ $3 \times 24 \mathrm{mg} /$ day: after 12 months, the average number of attacks declined from 7.6 per month to 4.4 in the low-dosage group and from 8.8 to 1.0 in the highdosage group in the group comparison [67]. If the patients do not respond sufficiently after 3 months on a dosage of $3 \times 48 \mathrm{mg} / \mathrm{day}$, the dose can be successively increased up to $480 \mathrm{mg} /$ day in individual cases [68]. At the present time, a multicenter, placebo-controlled, dose-finding study is being conducted $(3 \times 16$ vs. $3 \times 48 \mathrm{mg} /$ day) (EudraCT-Nr. 2005-000752-32; BMBF177zfyGT).

\section{VESTIBULAR PAROXYSMIA}

Vestibular paroxysmia is characterized by spontaneous, recurrent, short-lasting attacks of vertigo [71]. Constructive interference in the steady-state magnetic resonance imaging (CISS MRI) showed neurovascular cross-compression of the eighth nerve, particularly by the anterior inferior cerebellar artery [72], in more than 95\% of these patients.
Such vessel-nerve contacts are, however, also seen in healthy individuals, also well recognized for trigeminal neuralgia [73]. In trigeminal neuralgia, high-resolution diffusion tensor imaging revealed significant lower anisotropy and a higher apparent diffusion coefficient in the affected trigeminal root, which correlates with structural atrophic nerve changes [74]. A comparable study is not yet available for the eighth nerve.

The brief and frequent attacks of vertigo with postural imbalance respond to carbamazepine, even in low dosages [71]. A follow-up of 32 patients (mean follow-up time 31.3 months) proved not only the usefulness of the diagnostic criteria, but also the efficacy of this medical treatment [72]. Carbamazepine is currently the drug of first choice. There is limited evidence for the use of lamotrigine, baclofen, topiramate $[75,76]$, or nonantiepileptic drugs in trigeminal neuralgia [77] and for gabapentine in vestibular paroxysmia [78]. For medically refractive trigeminal neuralgia, nonablative microvascular decompression is still the preferred surgical approach [79], although a Cochrane analysis criticized the poor quality of clinical trials used to help in comparative decision-making regarding the best surgical procedure [80]. Microvascular decompression of the eighth nerve was shown earlier to be effective for the neurovascular compression of the 
eighth nerve [81] and also recently for a clinically well documented case of vestibular paroxysmia [82"].

Can central lesions mimic peripheral neurovascular compression syndromes? Trigeminal neuralgia and Lhermitte's phenomenon occur in multiple sclerosis and are classified as paroxysmal neuropathic pains caused by ectopic impulse generation along primary afferents [83]. So far, multiple sclerosis has not been described as a cause of vestibular paroxysmia because of demyelinating plaques at the root entry zone of the vestibular nerve. Carbamazepine-responsive paroxysmal dysarthria and ataxia in multiple sclerosis are different; they occur with midbrain lesions below the level of the red nucleus $[84,85]$.

\section{PERILYMPH FISTULA AND SUPERIOR CANAL DEHISCENCE SYNDROME}

The symptoms of perilymph fistula and its most important subtype SCDS are recurrent attacks of vertigo, dizziness, or imbalance induced by changes of pressure with or without hearing problems. The incidence and prevalence of perilymph fistulas are not known [1"]. The course of illness varies. As a rule, they resolve spontaneously and there are symptomfree periods of varying durations. It has been proposed that the presence of stable perilymph proteins is a specific marker of perilymph fistulas [86]; however, the diagnostic selectivity of these proteins is ambiguous [87].

So-called canal dehiscence most often involves the superior, rarely the lateral, or posterior semicircular canals [88]. The clinical diagnosis of SCDS must be confirmed by thin-slice computed tomography (CT) of the petrous bone, which shows an apical bony defect of the canal. High-resolution temporal bone CT, however, may demonstrate a false canal dehiscence [89]. Thus, it is necessary to combine radiological findings with the typical clinical signs and symptoms in order to confirm the diagnosis. Electrophysiologically cervical and ocular VEMP generally show a reduced sensory threshold $[90,91]$ in the affected (or worse ear). The length of the dehiscence found in patients who underwent surgical repair correlated with the maximal airbone gap, but not with clinical and electrophysiological variables [92].

Postoperative investigations indicate that there is some sensorineural hearing loss [93], transient impairment of balance measures, and a pathological head-impulse test in the plane of the affected canal [94]. Double-sided occlusion of the superior canal has been performed in patients with bilateral SCDS. Although a few of these patients complained in particular of oscillopsia, they were apparently satisfied with the improvement of the other symptoms [95]. The dehiscence syndrome also occurs in children, often at first only with auditory signs [96]. Conservative management is preferred for children.

\section{CONCLUSION}

For the most frequent forms of peripheral vestibular disorders (BPPV of the posterior, horizontal, or very rarely the anterior semicircular canal, Menière's disease, vestibular neuritis, vestibular paroxysmia, and SCDS), we have reliable diagnostic criteria which are currently being further improved by the Classification Committee of the Bárány Society. In Menière's disease, a new imaging technique with high-resolution MRI of the inner ear after transtympanic injection of gadolinium is a promising measure to visualize endolymphatic hydrops and thereby useful for a better understanding of its pathophysiology and the action of drugs used to treat this frequent disorder. There are still uncertainties in the treatment of Menière's disease, despite more than 3500 papers on this topic, acute vestibular neuritis, and vestibular paroxysmia which require state-of-the-art clinical trials.

\section{Acknowledgements}

The authors would like to thank Judy Benson for copyediting the manuscript.

\section{Conflicts of interest}

There are no conflicts of interest.

This work was supported by the Federal Ministry of Research and Education (BMBF) to the German Center for Vertigo and Balance Disorders (IFBLMU) (Grant No. 01E00901).

\section{REFERENCES AND RECOMMENDED}

\section{READING}

Papers of particular interest, published within the annual period of review, have been highlighted as:

- of special interest

a. of outstanding interest

Additional references related to this topic can also be found in the Current World Literature section in this issue (p. 107).

1. Brandt $T$, Dieterich $M$, Strupp M. Vertigo and dizziness - common complaints.

- 2nd ed. London: Springer; 2013.

A short clinically oriented textbook giving an overview of the current diagnostic criteria, pathophysiology, and treatment of the most frequent vestibular disorders.

2. Bisdorff $A$, von Brevern M, Lempert $T$, Newman-Toker DE. Classification of vestibular symptoms: towards an international classification of vestibular disorders. J Vestib Res 2009; 19:1-13.

3. Brandt $T$, Schautzer F, Hamilton D, et al. Vestibular loss causes hippocampal atrophy and impaired spatial memory in humans. Brain 2005; 128:27322741.

4. Zheng $\mathrm{Y}$, Balabhadrapatruni $\mathrm{S}$, Baek $\mathrm{JH}$, et al. The effects of bilateral vestibular loss on hippocampal volume, neuronal number, and cell proliferation in rats. Front Neurol 2012; 3:20.

5. Guinand N, Boselie F, Guyot JP, Kingma H. Quality of life of patients with bilateral vestibulopathy. Ann Otol Rhinol Laryngol 2012; 121:471-477. 
6. Kim S, Oh YM, Koo JW, Kim JS. Bilateral vestibulopathy: clinical characteristics and diagnostic criteria. Otol Neurotol 2011; 32:812-817.

7. Vital D, Hegemann SC, Straumann D, et al. A new dynamic visual acuity test to assess peripheral vestibular function. Arch Otolaryngol Head Neck Surg 2010; 136:686-691.

8. Guinand N, Pijnenburg M, Janssen M, Kingma H. Visual acuity while walking

- and oscillopsia severity in healthy subjects and patients with unilateral and bilateral vestibular function loss. Arch Otolaryngol Head Neck Surg 2012; 138:301-306.

Evaluation of the functional consequences on visual acuity in patients with chronic bilateral vestibulopathy.

9. Fujimoto $\mathrm{C}$, Murofushi T, Chihara Y, et al. Novel subtype of idiopathic bilateral vestibulopathy: bilateral absence of vestibular evoked myogenic potentials in the presence of normal caloric responses. J Neurol 2009; 256:1488-1492.

10. Szmulewicz DJ, Waterston JA, Halmagyi GM, et al. Sensory neuropathy as

-n part of the cerebellar ataxia neuropathy vestibular areflexia syndrome. Neurology $2011 ; 76: 1903-1910$.

Description of a frequent and clinically important entity characterized by the combination of central cerebellar and peripheral deficits.

11. Kirchner $\mathrm{H}$, Kremmyda $\mathrm{O}$, Hufner $\mathrm{K}$, et al. Clinical, electrophysiological, and MRI findings in patients with cerebellar ataxia and a bilaterally pathological head-impulse test. Ann N Y Acad Sci 2011; 1233:127-138.

12. McCall $A A$, Yates $B J$. Compensation following bilateral vestibular damage. Front Neurol 2011; 2:88.

13. Hillier SL, McDonnell M. Vestibular rehabilitation for unilateral peripheral vestibular dysfunction. Cochrane Database Syst Rev 2011; 2:CD005397.

14. Merfeld DM, Lewis RF. Replacing semicircular canal function with a vestibular implant. Curr Opin Otolaryngol Head Neck Surg 2012; 20:386-392.

15. Van de Berg R, Guinand N, Guyot JP, et al. The modified ampullar approach

I. for vestibular implant surgery: feasibility and its first application in a human with a long-term vestibular loss. Front Neurol 2012; 3:18.

The first clinical application of a vestibular implant as a new and promising techique to compensate for a bilateral vestibular deficit.

16. Cnyrim CD, Newman-Toker D, Karch $C$, et al. Bedside differentiation of vestibular neuritis from central 'vestibular pseudoneuritis'. J Neurol Neurosurg Psychiatry 2008; 79:458-460.

17. Kattah JC, Talkad AV, Wang DZ, et al. HINTS to diagnose stroke in the acute vestibular syndrome: three-step bedside oculomotor examination more sensitive than early MRI diffusion-weighted imaging. Stroke 2009; 40:35043510 .

18. Newman-Toker DE, Kattah JC, Alvernia JE, Wang DZ. Normal head impulse test differentiates acute cerebellar strokes from vestibular neuritis. Neurology 2008; 70:2378-2385.

19. Chen L, Lee W, Chambers BR, Dewey HM. Diagnostic accuracy of acute vestibular syndrome at the bedside in a stroke unit. J Neurol 2011; 258:855861.

20. Kim HA, Lee $\mathrm{H}$. Recent advances in central acute vestibular syndrome of a vascular cause. J Neurol Sci 2012; 321:17-22.

21. Arbusow V, Derfuss $T$, Held $K$, et al. Latency of herpes simplex virus type- 1 in human geniculate and vestibular ganglia is associated with infiltration of CD8 $^{+}$T cells. J Med Virol 2010; 82:1917-1920.

22. Roehm $P C$, Camarena $V$, Nayak $S$, et al. Cultured vestibular ganglion neurons demonstrate latent HSV1 reactivation. Laryngoscope 2011; 121:22682275.

23. Esaki S, Goshima F, Kimura H, et al. Auditory and vestibular defects induced by experimental labyrinthitis following herpes simplex virus in mice. Acta Otolaryngol 2011; 131:684-691.

24. Aw ST, Fetter M, Cremer PD, et al. Individual semicircular canal function in superior and inferior vestibular neuritis. Neurology 2001; 57:768-774.

25. Govender S, Colebatch JG. Ocular vestibular evoked myogenic potential (oVEMP) responses in acute vestibular neuritis. Clin Neurophysiol 2012; 123:1054-1055.

26. Shin BS, Oh SY, Kim JS, et al. Cervical and ocular vestibular-evoked myogenic potentials in acute vestibular neuritis. Clin Neurophysiol 2011.

27. Lin $\mathrm{CM}$, Young $\mathrm{YH}$. Identifying the affected branches of vestibular nerve in vestibular neuritis. Acta Otolaryngol 2011; 131:921-928.

28. Gianoli G, Goebel J, Mowry S, Poomipannit P. Anatomic differences in the lateral vestibular nerve channels and their implications in vestibular neuritis. Otol Neurotol 2005; 26:489-494.

29. Chihara $Y$, Iwasaki $S$, Murofushi $T$, et al. Clinical characteristics of inferior vestibular neuritis. Acta Otolaryngol 2012; 132:1288-1294.

30. Kim JS, Kim HJ. Inferior vestibular neuritis. J Neurol 2012; 259:1553-1560.

31. Brandt $\mathrm{T}$, Huppert $\mathrm{T}$, Hufner $\mathrm{K}$, et al. Long-term course and relapses of vestibular and balance disorders. Restor Neurol Neurosci 2010; 28:69-82.

32. Huppert $D$, Strupp $M$, Theil $D$, et al. Low recurrence rate of vestibular neuritis: a long-term follow-up. Neurology 2006; 67:1870-1871.

33. $\mathrm{Kim} \mathrm{YH}, \mathrm{Kim} \mathrm{KS}, \mathrm{Kim} \mathrm{KJ}$, et al. Recurrence of vertigo in patients with vestibular neuritis. Acta Otolaryngol 2011; 131:1172-1177.

34. Mandala M, Santoro GP, Awrey J, Nuti D. Vestibular neuritis: recurrence and incidence of secondary benign paroxysmal positional vertigo. Acta Otolaryngol 2010; 130:565-567.

35. Brandt T. Phobic postural vertigo. Neurology 1996; 46:1515-1519.

36. Strupp M, Zingler VC, Arbusow V, et al. Methylprednisolone, valacyclovir, or the combination for vestibular neuritis. N Engl J Med 2004; 351:354-361.
37. Karlberg ML, Magnusson M. Treatment of acute vestibular neuronitis with glucocorticoids. Otol Neurotol 2011; 32:1140-1143.

38. Goudakos JK, Markou KD, Franco-Vidal V, et al. Corticosteroids in the treatment of vestibular neuritis: a systematic review and meta-analysis. Otol Neurotol 2010; 31:183-189.

39. Fishman JM, Burgess C, Waddell A. Corticosteroids for the treatment of idiopathic acute vestibular dysfunction (vestibular neuritis). Cochrane Database Syst Rev 2011; CD008607.

40. Wegner I, van Benthem PP, Aarts MC, et al. Insufficient evidence for the effect of corticosteroid treatment on recovery of vestibular beuritis. Otolaryngol Head Neck Surg 2012; 147:826-831.

41. Von-Brevern $M$, Bertholon $P$, Fife $T$, et al. Benign paroxysmal positional vertigo - diagnostic criteria. J Vestib Res (in press).

A consensus document of the Committee for the International Classification of Vestibular Disorders of the Bárány Society.

42. Kim HA, Yi HA, Lee $\mathrm{H}$. Apogeotropic central positional nystagmus as a sole sign of nodular infarction. Neurol Sci 2012; 33:1189-1191.

43. Radtke $A$, von Brevern $M$, Neuhauser $H$, et al. Vestibular migraine: long-term

- follow-up of clinical symptoms and vestibulo-cochlear findings. Neurology 2012; 79:1607-1614.

A clinically very useful long-term follow-up study on the most frequent cause of recurrent spontaneous attacks of vertigo: vestibular migraine. It is also an important contribution to the differentiation between Menière's disease and vestibular migraine.

44. Fife TD, Iverson DJ, Lempert $T$, et al. Practice parameter: therapies for benign paroxysmal positional vertigo (an evidence-based review): report of the Quality Standards Subcommittee of the American Academy of Neurology. Neurology 2008; 70:2067-2074.

45. Yacovino DA, Hain TC, Gualtieri F. New therapeutic maneuver for anterior canal benign paroxysmal positional vertigo. J Neurol 2009; 256:1851-1855.

46. Vannucchi $P$, Pecci R. Pathophysiology of lateral semicircular canal paroxysmal positional vertigo. J Vestib Res 2010; 20:433-438.

47. Kim JS, Oh SY, Lee SH, et al. Randomized clinical trial for geotropic horizontal

- canal benign paroxysmal positional vertigo. Neurology 2012; 79:700-707.

A carefully performed clinical trial with immediate therapeutic consequences.

48. Nakashima T, Naganawa $S$, Sugiura $M$, et al. Visualization of endolymphatic hydrops in patients with Meniere's disease. Laryngoscope 2007; 117:415420.

49. Fiorino F, Pizzini FB, Beltramello $A$, et al. Reliability of magnetic resonance imaging performed after intratympanic administration of gadolinium in the identification of endolymphatic hydrops in patients with Meniere's disease. Otol Neurotol 2011; 32:472-477.

50. Nakashima $T$, Naganawa $S$, Teranishi $M$, et al. Endolymphatic hydrops revealed by intravenous gadolinium injection in patients with Meniere's disease. Acta Otolaryngol 2010; 130:338-343.

51. Tanigawa $T$, Tamaki $T$, Yamamuro $O$, et al. Visualization of endolymphatic hydrops after administration of a standard dose of an intravenous gadoliniumbased contrast agent. Acta Otolaryngol 2011; 131:596-601.

52. Gurkov R, Flatz W, Louza J, et al. In vivo visualization of endolymphatic hydrops in patients with Meniere's disease: correlation with audiovestibular function. Eur Arch Otorhinolaryngol 2011; 268:1743-1748.

53. Katayama $N$, Yamamoto $M$, Teranishi $M$, et al. Relationship between endolymphatic hydrops and vestibular-evoked myogenic potential. Acta Otolaryngol 2010; 130:917-923.

54. Fiorino F, Pizzini FB, Beltramello A, Barbieri F. MRI performed after intratympanic gadolinium administration in patients with Meniere's disease: correlation with symptoms and signs. Eur Arch Otorhinolaryngol 2011; 268:181187.

55. Grieve SM, Obholzer R, Malitz N, et al. Imaging of endolymphatic hydrops in Meniere's disease at $1.5 \mathrm{~T}$ using phase-sensitive inversion recovery: (1) demonstration of feasibility and (2) overcoming the limitations of variable gadolinium absorption. Eur J Radiol 2012; 81:331-338.

56. Claes G, Van den Hauwe L, Wuyts F, Van de Heyning P. Does intratympanic gadolinium injection predict efficacy of gentamicin partial chemolabyrinthectomy in Meniere's disease patients? Eur Arch Otorhinolaryngol 2012; 269:413-418.

57. Gurkov R, Flatz W, Keeser D, et al. Effect of standard-dose betahistine on endolymphatic hydrops: an MRI pilot study. Eur Arch Otorhinolaryngol 2012 [Epub ahead of print].

58. Pullens B, Giard JL, Verschuur HP, van Benthem PP. Surgery for Meniere's disease. Cochrane Database Syst Rev 2010; CD005395.

59. Thirlwall AS, Kundu S. Diuretics for Meniere's disease or syndrome. Cochrane Database Syst Rev 2006; 3:CD003599.

60. Strupp M, Thurtell MJ, Shaikh AG, et al. Pharmacotherapy of vestibular and ocular motor disorders, including nystagmus. J Neurol 2011; 258:12071222.

61. Stokroos R, Kingma $H$. Selective vestibular ablation by intratympanic gentamicin in patients with unilateral active Meniere's disease: a prospective, double-blind, placebo-controlled, randomized clinical trial. Acta Otolaryngol 2004; 124:172-175.

62. Postema RJ, Kingma CM, Wit HP, et al. Intratympanic gentamicin therapy for control of vertigo in unilateral Meniere's disease: a prospective, double-blind, randomized, placebo-controlled trial. Acta Otolaryngol 2008; 128: $876-880$ 
63. Pullens B, van Benthem PP. Intratympanic gentamicin for Meniere's disease or syndrome. Cochrane Database Syst Rev 2011; CD008234.

64. Garduno-Anaya MA, Couthino De TH, Hinojosa-Gonzalez R, et al. Dexamethasone inner ear perfusion by intratympanic injection in unilateral $\mathrm{Me}$ niere's disease: a two-year prospective, placebo-controlled, double-blind, randomized trial. Otolaryngol Head Neck Surg 2005; 133:285-294.

65. Phillips JS, Westerberg B. Intratympanic steroids for Meniere's disease or syndrome. Cochrane Database Syst Rev 2011; CD008514.

66. Casani AP, Piaggi $P$, Cerchiai $N$, et al. Intratympanic treatment of intractable

- unilateral Meniere disease: gentamicin or dexamethasone? A randomized controlled trial. Otolaryngol Head Neck Surg 2012; 146:430-437.

An important head-to-head comparison of two currently used treatments for Menière's disease, demonstrating the superiority of transtympanic gentamicin compared to steroids.

67. Strupp M, Hupert D, Frenzel C, et al. Long-term prophylactic treatment of attacks of vertigo in Meniere's disease - comparison of a high with a low dosage of betahistine in an open trial. Acta Otolaryngol 2008; 128:520-524.

68. Lezius F, Adrion C, Mansmann U, et al. High-dosage betahistine dihydrochloride between 288 and 480 mg/day in patients with severe Meniere's disease: a case series. Eur Arch Otorhinolaryngol 2011; 268:1237-1240.

69. Ihler F, Bertlich M, Sharaf K, et al. Betahistine exerts a dose-dependent effect

- on cochlear stria vascularis blood flow in Guinea pigs in vivo. PLoS One 2012; 7:e39086.

The first study showing a sigmoid dose-response curve for the effects of betahistine on cochlear blood flow, making this a likely mode of action in Menière's disease.

70. Strupp M, Cnyrim C, Brandt T. Vertigo and dizziness: treatment of benign paroxysmal positioning vertigo, vestibular neuritis and Menière's disease. In: Candelise L, editor. Evidence-based neurology - management of neurological disorders. Oxford: Blackwell Publishing; 2007. pp. 59-69.

71. Brandt T, Dieterich M. Vestibular paroxysmia: vascular compression of the eighth nerve? Lancet 1994; 343:798-799.

72. Hufner $\mathrm{K}$, Barresi D, Glaser $\mathrm{M}$, et al. Vestibular paroxysmia: diagnostic features and medical treatment. Neurology 2008; 71:1006-1014.

73. Peker $S$, Dincer $A$, Necmettin $P M$. Vascular compression of the trigeminal nerve is a frequent finding in asymptomatic individuals: 3-T MR imaging of 200 trigeminal nerves using 3D CISS sequences. Acta Neurochir (Wien) 2009; 151:1081-1088.

74. Leal PR, Roch JA, Hermier M, et al. Structural abnormalities of the trigeminal root revealed by diffusion tensor imaging in patients with trigeminal neuralgia caused by neurovascular compression: a prospective, double-blind, controlled study. Pain 2011; 152:2357-2364.

75. Zakrzewska JM, McMillan R. Trigeminal neuralgia: the diagnosis and management of this excruciating and poorly understood facial pain. Postgrad Med J 2011; 87:410-416.

76. Wang QP, Bai M. Topiramate versus carbamazepine for the treatment of classical trigeminal neuralgia: a meta-analysis. CNS Drugs 2011; 25:847-857.

77. Yang M, Zhou M, He L, et al. Nonantiepileptic drugs for trigeminal neuralgia. Cochrane Database Syst Rev 2011; CD004029.

78. Russell D, Baloh RW. Gabapentin responsive audiovestibular paroxysmia. J Neurol Sci 2009; 281:99-100.
79. Pollock BE. Surgical management of medically refractory trigeminal neuralgia. Curr Neurol Neurosci Rep 2012; 12:125-131.

80. Zakrzewska JM, Akram H. Neurosurgical interventions for the treatment of classical trigeminal neuralgia. Cochrane Database Syst Rev 2011; CD007312.

81. Jannetta PJ, Moller MB, Moller AR. Disabling positional vertigo. N Engl J Med 1984; 310:1700-1705.

82. Strupp M, Stuckrad-Barre S, Brandt T, Tonn JC. Compression of the eighth

- cranial nerve causes vestibular paroxysmia. Neurology (in press).

A convincing description of a compression of the eighth nerve in a clinically proven case of vestibular paroxysmia, demonstrating in parallel a clear benefit from surgery.

83. Truini A, Barbanti P, Pozzilli C, Cruccu G. A mechanism-based classification of pain in multiple sclerosis. J Neurol 2012. [Epub ahead of print]

84. Marcel C, Anheim M, Flamand-Rouviere C, et al. Symptomatic paroxysmal dysarthria-ataxia in demyelinating diseases. J Neurol 2010; 257:13691372.

85. Li Y, Zeng C, Luo T. Paroxysmal dysarthria and ataxia in multiple sclerosis and corresponding magnetic resonance imaging findings. J Neurol 2011; 258:273-276.

86. Shiiba $K$, Shindo $S$, Ikezono $T$, et al. Cochlin expression in the rat perilymph during postnatal development. Acta Otolaryngol 2012; 132:1134-1139.

87. Bachmann-Harildstad G, Stenklev NC, Myrvoll E, et al. Beta-trace protein as a diagnostic marker for perilymphatic fluid fistula: a prospective controlled pilot study to test a sample collection technique. Otol Neurotol 2011; 32:7-10.

88. Chien WW, Carey JP, Minor LB. Canal dehiscence. Curr Opin Neurol 2011; 24:25-31.

89. Sequeira SM, Whiting BR, Shimony JS, et al. Accuracy of computed tomography detection of superior canal dehiscence. Otol Neurotol 2011; 32:1500-1505.

90. Thabet EM, Abdelkhalek A, Zaghloul H. Superior semicircular canal dehiscence syndrome as assessed by oVEMP and temporal bone computed tomography imaging. Eur Arch Otorhinolaryngol 2012; 269: $1545-1549$

91. Niesten ME, McKenna MJ, Herrmann BS, et al. Utility of cVEMPs in bilateral superior canal dehiscence syndrome. Laryngoscope 2012. [Epub ahead of print]

92. Chien WW, Janky K, Minor LB, Carey JP. Superior canal dehiscence size: multivariate assessment of clinical impact. Otol Neurotol 2012; 33: 810-815.

93. Ward BK, Agrawal $Y$, Nguyen E, et al. Hearing outcomes after surgical plugging of the superior semicircular canal by a middle cranial fossa approach. Otol Neurotol 2012; 33:1386-1391.

94. Janky KL, Zuniga MG, Carey JP, Schubert M. Balance dysfunction and recovery after surgery for superior canal dehiscence syndrome. Arch Otolaryngol Head Neck Surg 2012; 138:723-730.

95. Agrawal $\mathrm{Y}$, Minor LB, Schubert MC, et al. Second-side surgery in superior canal dehiscence syndrome. Otol Neurotol 2012; 33:72-77.

96. Lee GS, Zhou G, Poe D, et al. Clinical experience in diagnosis and management of superior semicircular canal dehiscence in children. Laryngoscope $2011 ; 121: 2256-2261$. 